\title{
Cognitive Radio Operation under Directional Primary Interference and Practical Path Loss Models
}

\author{
Kentaro NISHIMORI $^{\dagger \mathrm{a})}$, Senior Member, Rocco DI TARANTO ${ }^{\dagger \dagger}$, Nonmember, Hiroyuki YOMO ${ }^{\dagger \dagger, \dagger \dagger \dagger}$, Member $^{\dagger}$ \\ and Petar POPOVSKI ${ }^{\dagger \dagger}$, Nonmember
}

\begin{abstract}
SUMMARY This paper discusses the possibility of deploying a shortrange cognitive radio (secondary communication system) within the service area of a primary system. Although the secondary system interferes with the primary system, there are certain locations in the service area of the primary system where the cognitive radio can reuse the frequency of the primary system without causing harmful interference to it and being disturbed by the primary system. These locations are referred to as having a spatial opportunity for communications in the secondary system, since it can reuse the frequency of the primary system. Simulation results indicate that the antenna gain, beamwidth, and propagation path loss greatly affect the spatial opportunity of frequency reuse for the secondary users. The results show that spatial spectrum reuse can be significantly increased when the primary system users are equipped with directional antennas. An important component in this study is the heterogeneous path loss model, i.e., the path loss model within the primary system is different from the model used to calculate the interference between the primary and the secondary systems. Our results show that the propagation models corresponding to the actual antenna heights in the primary/secondary system can largely impact the possibilities for spectrum reuse by the cognitive radios.

key words: cognitive radio, spatial opportunity, directional antenna, heterogeneous propagation path loss conditions, target CIR, antenna height
\end{abstract}

\section{Introduction}

Due to the immense popularity of mobile phones and wireless LAN systems, wireless communication systems have been challenged with increasing demand to offer the target quality of service with a limited frequency bandwidth. Traditional and common approaches to address this challenge have been to increase the number of bits that can be transmitted per unit time and frequency, resulting in high capacity with a given frequency bandwidth. However, it was shown by a recent report by the Federal Communication Commission (FCC) that a large part of the licensed spectrum is not utilized most of the time and space [1]. Hence, we can dramatically improve the frequency utilization if we allow a wireless node (often called a secondary system or cognitive radio) to access dynamically the spectrum that is under-utilized in space/time by the licensed system (often

\section{Manuscript received August 30, 2010.}

Manuscript revised December 17, 2010.

${ }^{\dagger}$ The author is with the Faculty of Engineering, Niigata University, Niigata-shi, 950-2102 Japan.

${ }^{\dagger}$ The authors are with the Department of Electronic Systems, Aalborg University Niels Jernes Vej 12, DK-9220 Aalborg, Denmark.

${ }^{\dagger \dagger}$ The author is with the Faculty of Engineering Science, Kansai University, Suita-shi, 564-8680 Japan.

a)E-mail: nishimori@m.ieice.org

DOI: 10.1587/transcom.E94.B.1243 called the primary system) [2], [3].

In this scenario, it is very important to investigate how the secondary or cognitive users can communicate inside the service area of the primary system [4]. When co-existence between the primary and secondary systems is considered, the secondary system is not allowed to cause harmful interference to the primary system. Hence, the secondary system must quantify and bound its interference to the primary system. A model was proposed to achieve such co-existence between a broadcast system such as TV with a large transmission range and a system with a small cell size such as a Wi-Fi [5]. In this study, the maximum transmit power that is required for the secondary system is formulated as a function of the actual antenna/amplifier gains and propagation path loss [5].

The primary systems are usually assumed to use not only omni-directional antennas but also directional antennas in order to increase the service area. When considering a scenario where primary and/or cognitive systems are equipped with directional antennas, the precise antenna pattern must be considered to calculate the interference between the two coexisting systems. This is because the interference range decreases as the direction for the receiver moves away from the direction of the main beam on the directional antenna. Moreover, when directional antennas are considered, the interference model should be dependent on the antenna gain, the beamwidth, as well as the beam geometry. The use of directional antennas/smart antenna/beamforming at the primary/secondary transmitter was discussed in references [6]-[8]. However, references [6] and [7] do not deal with the directional antennas on the primary system, but only on the secondary system. On the other hand, reference [8] deals with the smart antenna not only for the secondary system but also for the primary system. However, in the previous works, the influence of the beamwidth at the primary transmitter has not been considered to study the spatial opportunity for cognitive radio systems.

Since the primary and secondary systems have different features and usage scenarios, deployment conditions for the primary and secondary systems are principally different. For example in a long-range primary system, the base stations are located on the rooftops of buildings. On the other hand, secondary transceivers are placed at various locations (indoor/outdoor, high/low antenna heights, urban/rural environment, etc.). Thus, the differences between the deploy- 
ment conditions of the primary and secondary systems give rise to differences in the path loss for a link within the primary system and the path loss for the interference from the primary to secondary system (and vice versa).

In this paper, we investigate spatial opportunity when a short-range secondary system should coexist with a largerange primary system that employs directional antenna [9]. We derive the spatial availability as the function of antenna and propagation's parameters and clarify that the spatial opportunities of cognitive systems could be significantly enhanced when assuming the directional antenna with narrow beamwidth for the primary systems [9].

This paper also derives the spatial opportunity when the heterogeneous propagation path loss conditions are considered between the communication link within the primary system and the interference from the primary to secondary system [10]. To achieve this, we introduce the propagation loss model from [11]. This model can be utilized even if antenna heights of both the transmitter and receiver are varied. In other words, all the heterogeneous path loss conditions in our target scenarios can be modeled using [11]. The spatial opportunity for the secondary systems is evaluated when the several scenarios are considered in actual systems with various antenna heights of the primary and secondary systems.

This paper focuses on new aspects compared to the previous studies:

- We consider and evaluate two types of inter-system interference: Interference from the primary to secondary system and, vice versa, from the secondary to primary system.

- In our previous studies [9], [10], we assumed that the receiver is always positioned at the edge of the service area. However, the Carrier to Interference Ratio (CIR) changes significantly according to the relationship of positions between the transmitter and receiver as well as between the source of interference and receiver. In this paper, we define that the primary/secondary users are located uniformly inside the service area.

- In addition to the heterogeneous conditions for path loss, we also consider short-term fading effects.

- In this paper, we consider multi-user scenarios that generally occur in commercial systems.

The rest of the paper is organized as follows. In Sect. 2, we describe the target system and the motivation for the research. Section 3 derives the spatial opportunity for cognitive radio systems when we consider heterogeneous propagation loss conditions where different path loss coefficients are used for the link between primary users and the interference from the primary to secondary transceivers. In Sect. 4, the spatial opportunity for the secondary system with numerical results is clarified when various antenna heights for the primary and secondary systems are assumed. Finally, Sect. 5 concludes the paper and discusses possible future work.

\section{Target Scenario and Path Loss Model}

\subsection{Target Scenario}

Figure 1 shows an example of the target scenario. The characteristics of the systems with large and small areas significantly differ from each other. The strategy discussed herein takes advantage of the features of both primary and secondary systems in order to reach an efficient state of coexistence [4], [5], [9], [10].

In the figure, we assume bi-directional communication between the base station (BS) and the subscriber station (SS) for a primary system, where both BS and SS can transmit/receive the signals, respectively. As can be seen in Fig. 1, Fixed Wi-MAX can be considered as an actual example of such a primary system. The BS antenna is positioned at a high location. The SS antenna is placed at either a high or low location. Both the BS and SS use directional antennas to enlarge the respective service areas. Therefore, the primary communicable range is maximum in the direction of the main beam while it gradually decreases in the other directions. This provides an opportunity to deploy cognitive devices in the directions of the side lobe [9].

We consider a secondary system that has a much shorter communication range than that for the primary system and attempts to reuse the frequency band assigned to the primary system. Since communications are confined to a local area, such a secondary system has a high potential to find flexibly available space within the service area of the primary system. Examples of such short range systems are the indoor or outdoor WLANs. In this paper, we introduce path loss model that can be applied to both indoor and outdoor scenarios of a secondary system. For the secondary short-range systems, it is reasonable to assume use of omnidirectional antennas.

We assume that the secondary users know the antenna gain, amplifier gain and noise figure in both primary and secondary systems. Therefore, before starting their communication, the secondary transmitters can estimate their interference toward the primary receivers by using the received power (detected during primary transmission) from a primary transmitter toward the secondary receivers. The actual method for the estimation of the interference power from the secondary transmitters toward a primary receiver is detailed in [12]. The method introduced in [12] counts on the channel reciprocity which can be violated when considering mobility as well as different interference conditions at two end-points. The lack of channel reciprocity results in the degradation of spatial opportunity as the secondary transmitter underestimates the interference and starts transmission, which consequently violates the target quality at primary receiver or overestimates the interference and does not start transmission itself. In order to minimize such degradation, more precise method to estimate interference is required. We consider the development of methods to estimate exact channel state information among nodes as a comple- 


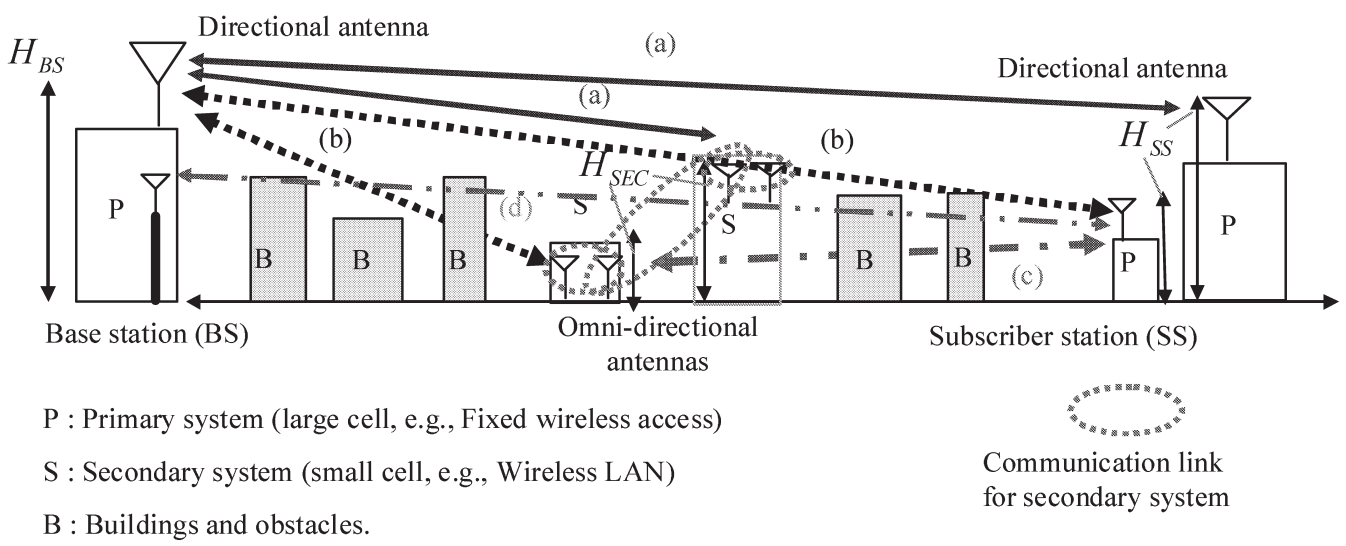

Fig. 1 Example of target scenario in cognitive radio system.

mentary work to this paper, and keep it for our future work.

\subsection{Propagation Path Loss Model Considering Heteroge- neous Path Loss Condition}

The propagation path loss significantly affects the spatial opportunity for cognitive radio systems [5]. Several propagation path loss models are proposed for indoor and outdoor environments in [13]-[18]. A general expression for the path loss model $L(d)(d$ : distance between the transmitter and receiver) can be written as follows:

$$
L(d)=\alpha \cdot \log _{10}(d)-\beta[\mathrm{dB}] \quad \alpha, \beta \in \mathrm{R},
$$

where $\alpha$ ranges from 20 to $40 \mathrm{~dB}$, while $\beta$ is determined by various factors such as antenna height, frequency, building structure and width of the road etc. [13]-[18].

In this paper we consider the following four different path loss conditions, corresponding to (a)-(d) shown in Fig. 1 where the primary transmitter is set higher than the surrounding buildings:

(a) Secondary $\mathrm{Tx} / \mathrm{Rx}$ and Primary $\mathrm{Rx}$ are set higher than the surrounding buildings

(b) Secondary $\mathrm{Tx} / \mathrm{Rx}$ is set higher and Primary $\mathrm{Rx}$ is set lower than the surrounding buildings

(c) Secondary $\mathrm{Tx} / \mathrm{Rx}$ and Primary Rx are set lower than the surrounding buildings

(d) Others

Looking at the target scenario shown in Fig. 1, we notice that the propagation path losses are obviously different among the BS to/from the SS, the BS to/from the secondary systems, and the SS to/from the secondary systems, because different antenna height greatly affects on the propagation characteristics. Hence, a propagation path loss model taking into account different antenna heights is needed.

Conventional path loss models in [13]-[17] can deal only with condition (b) described above, where the antenna heights of BS and SS are high and low, respectively. This is because the propagation coefficient, $\alpha$ is denoted as a function on antenna height of the BS. The antenna height on the SS is low in the conventional path loss models. On the other
Table 1 Path loss model with heterogeneous path loss conditions.

\begin{tabular}{|l|}
\hline$L(d)=\left[51-8 \log _{10}\left(H_{\mathrm{Tx}} H_{\mathrm{Rx}}\right)\right] \log _{10}(d)+8.4 \log _{10}\left(H_{\mathrm{Tx}} H_{\mathrm{Rx}}\right)$ \\
$\quad+20 \log _{10}\left(\mathrm{f}_{\mathrm{c}} / 2.2\right)+14$ \\
\hline$L(d):$ Path $\operatorname{loss}_{(\mathrm{dB})}$ \\
$d \quad:$ Distance between transmitter and receiver $(200-3000[\mathrm{~m}])$ \\
$H_{T x}:$ Antenna height at the transmitter $(3.5-30[\mathrm{~m}])$ \\
$H_{R x}:$ Antenna height at the receiver $(1.5-30[\mathrm{~m}])$ \\
$\mathrm{f}_{\mathrm{c}} \quad:$ Frequency $(2 \mathrm{GHz}$ band $)$ \\
$\alpha \quad: 51-8 \log _{10}\left(H_{\mathrm{Tx}} H_{\mathrm{Rx}}\right)$ \\
$\beta \quad:-8.4 \log _{10}\left(H_{\mathrm{Tx}} H_{\mathrm{Rx}}\right)-20 \log _{10}\left(\mathrm{f}_{\mathrm{c}} / 2.2\right)-14$ \\
\hline
\end{tabular}

Table 2 Path loss model for indoor scenarios.

\begin{tabular}{|c|c|}
\hline$\alpha$ & $\beta$ \\
\hline$n$ & $20 \log _{10}[f]_{\mathrm{GHz}}+L_{f}+20 \log _{10}(4 \pi \lambda / c)+180$ \\
\hline
\end{tabular}

hand, we found a path loss model which encompasses (a)(d) [11], called Ichitsubo's model. Table 1 presents parameters of Ichitsubo's model. As can be seen in Table 1, Ichitsubo's model can be used for all four cases shown in Fig. 1, because $\alpha$ and $\beta$ are functions of the antenna heights of the transmitter and receiver.

This model was obtained considering many measurements, which were carried out with $2 \mathrm{GHz}$ band in urban areas in Japan. Moreover, the path losses obtained by the measurements and the equation in Table 1 agree well with each other, when the transmissions of Tx 32 [m]-Rx 32 [m], Tx 32 [m]-Rx 15 [m], and Tx 32 [m]-Rx 2 [m] are considered [11]. Here, Tx A [m]-Rx B [m] represent the antenna heights of the transmitter and receiver at A and B [m], respectively. Although none of parameters in Table 1 directly represent the effect of buildings and obstacles, parameters $\alpha$ and $\beta$ are certainly related to them. In fact, $\alpha$ and $\beta$ are function of the Tx and Rx antenna heights, and therefore they are affected by the presence/absence of obstacles and buildings. Therefore, since this model covers all the possible scenarios analyzed in this paper, we use Ichitsubo's model hereafter.

In this paper, for indoor scenarios the model in [18] is used. The values of $\alpha$ and $\beta$ used in indoor path loss model are shown in Table 2. Constants $n$ and $L_{f}$ represent heuristic parameters used to take into consideration the wall, ceiling, and floor attenuations. A typical value of $n$ in an 
office environment is 30 , whereas $L_{f}$ generally takes values between 10 to $20 \mathrm{~dB}$ per wall [18].

\section{Spatial Opportunity for Cognitive Radio Systems}

\subsection{Definition of Spatial Opportunity}

In this section, we give a formal definition for spatial opportunity in order to quantify the opportunity for secondary users to reuse frequency resources when located within the primary service area. In this paper, we deal with the case when the secondary system attempts to communicate during a transmission in the primary system. This case is more interesting than the uplink case because generally more resources in the time and frequency domains are assigned in the downlink channel than in the uplink channel. Moreover, the interference to the primary system is more critical during a downlink transmission. In order to achieve co-existence, we must consider two types of interference:

$\mathrm{I}_{1}$ Interference from the Primary Transmitter (PT) to the Secondary Receiver (SR)

$\mathrm{I}_{2}$ Interference from the Secondary Transmitter (ST) to the Primary Receiver (PR)

In this section and for rest of the paper, the BS and SS in Fig. 1 are regarded as the PT and PR, respectively.

\subsection{Flow to Obtain Spatial Opportunity}

Figure 2 shows the graphic representation of the algorithm (i.e., the flowchart) we have used to calculate the spatial opportunity. As it can be seen, the communicable areas for the primary and secondary systems are determined in Step

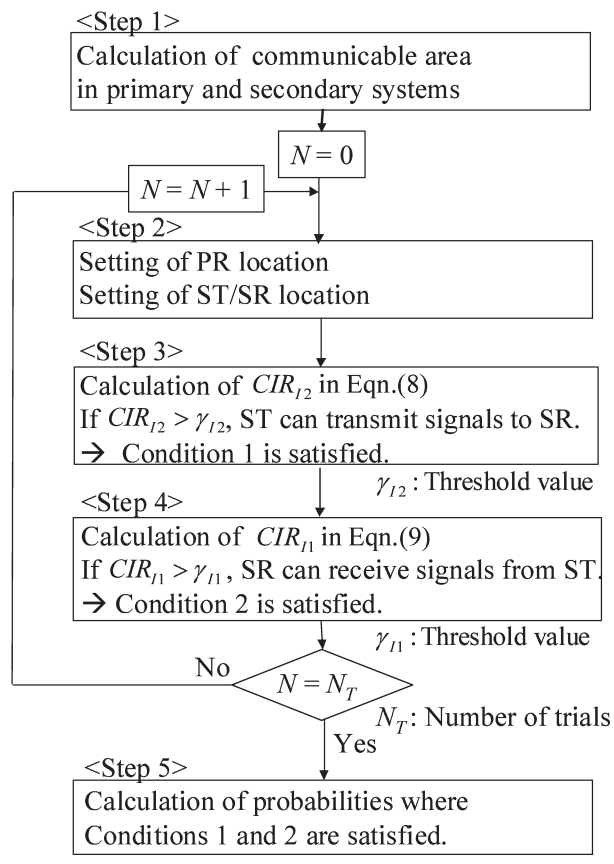

Fig. 2 Flow chart for obtaining spatial opportunity.
1 in the flowchart. The communicable area is defined as the area in which the signal from a given transmitter reaches its intended receiver with an average Carrier-to-Noise-Ratio (CNR) above a threshold, which is chosen according to actual system requirements. The primary (secondary) communicable area is the area around the PT (ST) where the PR (SR) is possibly located. In our evaluation we assume that secondary communicable area is always located inside the primary communicable area.

Figure 3 shows the primary communicable area when the primary users are equipped with omni-directional (Fig. 3(a)) and directional (Fig. 3(b)) antennas. $d_{\mathrm{Tx}, \mathrm{Rx}}^{\max }$ represents the distance between the Tx and Rx when the receiver is located at the edge of the service area in Fig. 3. Since secondary users are equipped with omnidirectional antennas, Fig. 3(a) represents also the secondary communicable area. When considering omni-directional antenna, amplifier gain and path loss, the communicable area is circular. Moreover, when the primary $\mathrm{Tx}$ and $\mathrm{Rx}$ are equipped with directional antennas, the communicable area is decided assuming that they generate their main beams toward each other. Hence, as shown in Fig. 3(b), the primary communicable area is circular, but with a larger radius.

Given the communicable area, we can determine the minimum received power required to achieve target CNR at the edge of the service area, $R_{\mathrm{Tx}}^{\min \mathrm{Rx}}$ as follows (in general, the following is valid for any pair of $\mathrm{Tx}$ and $\mathrm{Rx}$ within the

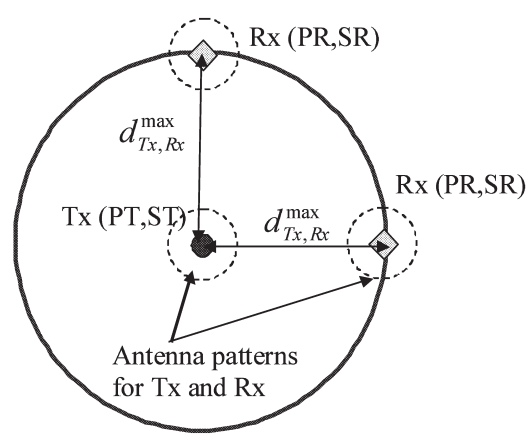

(a) When employing omni-directional antennas (for PT/PR, ST/SR).

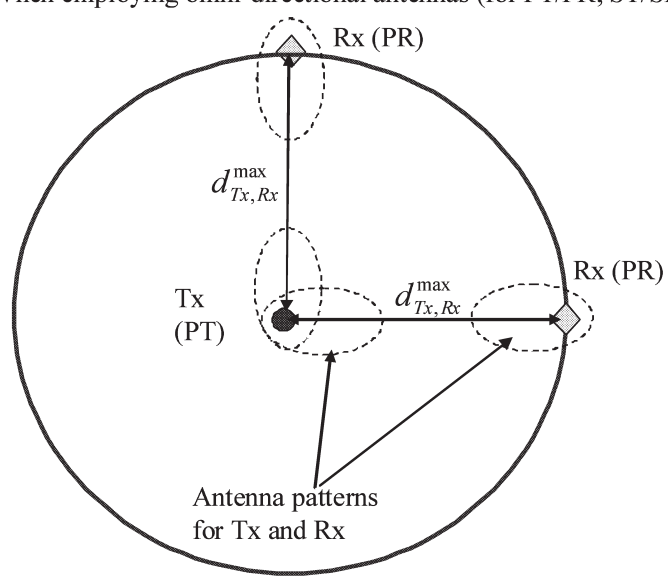

(b) When employing directional antennas (for PT/PR).

Fig. 3 Definition of communicable area. 


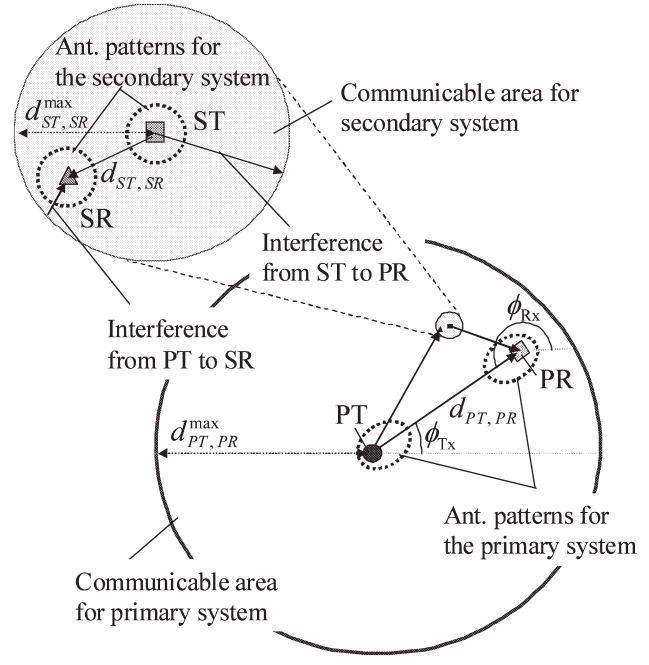

Fig. 4 Geometrical relationship among PT, PR, ST, SR.

primary and secondary communicable area):

$$
\begin{aligned}
R_{\mathrm{Tx}, \mathrm{Rx}}^{\min }= & G_{\mathrm{Tx}, \mathrm{HPA}}+G_{\mathrm{Tx}, \mathrm{ANT}}+F_{\mathrm{Tx}, \mathrm{BW}}\left(\phi_{\mathrm{Tx}}\right) \\
& -L\left(d_{\mathrm{Tx}, \mathrm{Rx}}^{\max }\right)+G_{\mathrm{Rx}, \mathrm{ANT}} \\
& +F_{\mathrm{Rx}, \mathrm{BW}}\left(\phi_{\mathrm{Rx}}\right)[\mathrm{dBm}], \\
L\left(d_{\mathrm{Tx}, \mathrm{Rx}}^{\max }\right) & =\alpha \log _{10}\left(d_{\mathrm{Tx}, \mathrm{Rx}}^{\max }\right)-\beta[\mathrm{dB}], \\
\mathrm{CNR}_{\mathrm{Tx}, \mathrm{Rx}} & =R_{\mathrm{Tx}, \mathrm{Rx}}^{\min }-G_{\mathrm{Rx}, \mathrm{LNA}},
\end{aligned}
$$

where $L\left(d_{\mathrm{Tx}, \mathrm{Rx}}^{\max }\right)$ is determined by the parameter in Tables 1 and/or 2. $G_{\mathrm{Tx}}$, ANT and $G_{\mathrm{Rx}}$, ANT are the gains of antennas for Tx/Rx, respectively. $G_{\mathrm{Tx}, \mathrm{HPA}}$ is the transmit power for Tx. $F_{\mathrm{Tx}, \mathrm{BW}}\left(\phi_{\mathrm{Tx}}\right) / F_{\mathrm{Rx}, \mathrm{BW}}\left(\phi_{\mathrm{Rx}}\right)$ is the normalized response of antenna pattern for direction $\phi_{\mathrm{Tx}} / \phi_{\mathrm{Rx}}$ in the horizontal plane at Tx and Rx, respectively. BW is the value of $3 \mathrm{~dB}$ beamwidth. $\phi_{\mathrm{Tx}}$ and $\phi_{\mathrm{Rx}}$ express the main beam direction at the Tx and Rx, respectively. When considering the primary system, since the PT and PR direct their main beams toward each other (at least in the ideal case) $F_{\mathrm{Tx}, \mathrm{BW}}\left(\phi_{\mathrm{Tx}}\right)$ and $F_{\mathrm{Rx}, \mathrm{BW}}\left(\phi_{\mathrm{Rx}}\right)$ are always $0 \mathrm{~dB}$. On the other hand, when considering the secondary system, since we assume that both $\mathrm{Tx}$ and $\mathrm{Rx}$ are equipped with omnidirectional antennas, $F_{\mathrm{Tx}, \mathrm{BW}=360^{\circ}}(\cdot)$ and $F_{\mathrm{Rx}, \mathrm{BW}=360^{\circ}}(\cdot)$ are always $0 \mathrm{~dB} . \mathrm{CNR}_{\mathrm{Tx}, \mathrm{Rx}}^{\min }$ denotes the CNR at the edge of service area. $G_{\mathrm{Rx} \text {, LNA }}$ is the noise power at the receiver. Based on all the previous assumptions, and using relationships in Eqs. (2) and (3), the communicable area for the primary or secondary system, $S_{\mathrm{C}}$ can be determined as follows:

$$
\begin{aligned}
& S_{\mathrm{C}}=\pi\left(d_{\mathrm{Tx}, \mathrm{Rx}}^{\max }\right)^{2}, \\
& d_{\mathrm{Tx} \mathrm{Rx}}^{\max }=10^{X_{\mathrm{C}} / \alpha}, \\
& X_{\mathrm{C}}=G_{\mathrm{Tx}, \mathrm{HPA}}+G_{\mathrm{Tx}, \mathrm{ANT}}+G_{\mathrm{Rx}, \mathrm{ANT}} \\
& \quad+G_{\mathrm{Rx}, \mathrm{LNA}}+\beta-R_{\mathrm{Tx}, \mathrm{Rx}}^{\min } .
\end{aligned}
$$

In Step 2, the position of the PR is determined inside the communicable area of the primary system, which is calculated in Step 1. Figure 4 shows the geometrical relationship among the PT, PR, ST and SR. Depending on the position of PR, the distance between the PT and PR, $d_{\mathrm{PT}, \mathrm{PR}}$ and the beam direction with maximum power for the PT/PR, $\phi_{\mathrm{PT}} / \phi_{\mathrm{PR}}$ are calculated, respectively. Concerning the secondary system, the ST is first located randomly within the communicable area of the primary system. Here, the ST is located so that the secondary communicable area is located inside the primary communicable area. The position of the SR is randomly selected within the communicable area of the secondary system, which is determined based on the position of ST. Then, the distance between the ST and SR, $d_{\mathrm{ST}, \mathrm{SR}}$ is calculated.

In Step 3 and Step 4, we examine whether the secondary transmissions are possible or not based on CIR (Carrier to Interference power Ratio) quality. The secondary transmission is impossible if a) they cause harmful interference to the primary system (Step 3) and/or b) the target quality at the secondary receiver is not satisfied due to the primary interference (Step 4). In order to quantify the spatial opportunity, we must define the target CIR, which represents the power ratio of the desired signal to interference that must be satisfied at the receiver. In this paper, not only path loss effects but also small-term fading (i.e., Rayleigh fading) effects are considered.

In Step 3, we check CIR at PR, $\mathrm{CIR}_{\mathrm{I}_{2}}$. $\mathrm{CIR}_{\mathrm{I}_{2}}$ can be obtained as the power ratio of the desired signal for the PT, $R_{\mathrm{PT}, \mathrm{PR}}$ to the interference from the $\mathrm{ST}$ at the PR, $R_{\mathrm{ST}, \mathrm{PR}}$. $\mathrm{CIR}_{\mathrm{I}_{2}}$ is obtained as follows:

$$
\begin{aligned}
\mathrm{CIR}_{\mathrm{I}_{2}}= & R_{\mathrm{PT}, \mathrm{PR}}-R_{\mathrm{ST}, \mathrm{PR}} \\
= & G_{\mathrm{PT}, \mathrm{HPA}}+G_{\mathrm{PT}, \mathrm{ANT}} \\
& -L\left(d_{\mathrm{PT}, \mathrm{PR}}\right)-L_{\mathrm{SF}, \mathrm{PT}, \mathrm{PR}}(t) \\
& -G_{\mathrm{ST}, \mathrm{HPA}}-G_{\mathrm{ST}, \mathrm{ANT}} \\
& +L\left(d_{\mathrm{ST}, \mathrm{PR}}\right)+L_{\mathrm{SF}, \mathrm{ST}, \mathrm{PR}}(t) \\
& -F_{\mathrm{PR}, \mathrm{BW}}\left(\theta_{\mathrm{ST}, \mathrm{PR}}\right)[\mathrm{dB}],
\end{aligned}
$$

where $\theta_{\mathrm{ST}, \mathrm{PR}}$ is the direction from the ST to the PR. Note that the effect of $F_{\mathrm{PR}, \mathrm{BW}}\left(\theta_{\mathrm{ST}, \mathrm{PR}}\right)$ must be considered unlike the case of the desired signal. While the PR always directs its main beam toward the PT to maximize its desired signal level, it is not directed toward ST. Therefore, $F_{\mathrm{PR}, \mathrm{BW}}\left(\theta_{\mathrm{ST}, \mathrm{PR}}\right)$ takes into account the antenna response of the PR in the direction of the ST. $L_{\mathrm{SF}}(t)$ denotes the smallscale fading effect. Coming back to our algorithm (Fig. 2, Step 3), we define a flag variable, $N_{C 2}(i)$, which for each trial $i$ in our evaluation, assumes value 1 if $\mathrm{CIR}_{\mathrm{I}_{2}}$ is greater than a given threshold, and 0 otherwise. $N_{T}$ represents the total number of trials in our evaluation.

In Step 4, we calculate CIR at SR, $\mathrm{CIR}_{\mathrm{I}_{1}}$ where the desired and interfering signals are from the $\mathrm{ST}$ and from the PT, respectively. $\mathrm{CIR}_{\mathrm{I}_{1}}$ can be obtained as the power ratio of the desired signal, $R_{\mathrm{ST} \text {, SR }}$, to the interference at the $\mathrm{SR}$, $R_{\mathrm{PT}, \mathrm{SR}} \cdot \mathrm{CIR}_{\mathrm{I}_{1}}$ is expressed as

$$
\begin{aligned}
\mathrm{CIR}_{\mathrm{I}_{1}}= & R_{\mathrm{ST}, \mathrm{SR}}-R_{\mathrm{PT}, \mathrm{SR}} \\
= & G_{\mathrm{ST}, \mathrm{HPA}}+G_{\mathrm{ST}, \mathrm{ANT}} \\
& -L\left(d_{\mathrm{ST}, \mathrm{SR}}\right)-L_{\mathrm{SF}, \mathrm{ST}, \mathrm{SR}}(t) \\
& -G_{\mathrm{PT}, \mathrm{HPA}}-G_{\mathrm{PT}, \mathrm{ANT}}
\end{aligned}
$$




$$
\begin{aligned}
& +L\left(d_{\mathrm{PT}, \mathrm{SR}}\right)+L_{\mathrm{SF}, \mathrm{PT}, \mathrm{SR}}(t) \\
& -F_{\mathrm{PT}, \mathrm{BW}}\left(\theta_{\mathrm{PT}, \mathrm{SR}}\right)[\mathrm{dB}],
\end{aligned}
$$

where $\theta_{\mathrm{PT}, \mathrm{SR}}$ is the direction from the PT to the SR. The effect of $F_{\mathrm{PT}, \mathrm{BW}}\left(\theta_{\mathrm{PT}, \mathrm{SR}}\right)$ must be considered like the calculation of $\mathrm{CIR}_{\mathrm{I}_{2}}$. Similarly as in Step 3, in our calculation (Fig. 2, Step 4), we have defined a flag variable $N_{C 1}(i)$, for each trial $i$, which assumes value 1 if $\mathrm{CIR}_{\mathrm{I}_{1}}$ is greater than a given threshold, or 0 otherwise.

As it is shown in Fig. 2, the loop from Step 2 to Step 4 is repeated $N_{T}$ times in our calculations. For each trial, the positions of PR, ST and SR are randomly generated as described above. In Step 5 it is calculated the probability that both $\mathrm{CIR}_{\mathrm{I}_{1}}$ and $\mathrm{CIR}_{\mathrm{I}_{2}}$ are greater than the respective thresholds defined above. We define this probability (ratio between the number of trials satisfying these two conditions and the total number of trials, $N_{T}$ in simulation) as the spatial opportunity for secondary system in our work. In our calculations, we first obtain the probability that either the condition on $\mathrm{CIR}_{\mathrm{I}_{1}}$ (Eq. (9)) or $\mathrm{CIR}_{\mathrm{I}_{2}}$ (Eq. (8)) is satisfied (by using Eqs. (10) and (11)); then we define the spatial opportunity as the joint probability that these two conditions are satisfied in Eq. (12):

$$
\begin{aligned}
\operatorname{Pr}_{1}= & \frac{\sum_{i}^{N_{T}} N_{C 1}(i)}{N_{T}} \times 100[\%], \\
\operatorname{Pr}_{2}= & \frac{\sum_{i}^{N_{T}} N_{C 2}(i)}{N_{T}} \times 100[\%], \\
\operatorname{Pr}_{\text {Total }}= & \frac{\left.\sum_{i}^{N_{T}} \min \left[N_{C 1}(i), N_{C 2}(i)\right)\right]}{N_{T}} \times 100[\%],
\end{aligned}
$$

where $P r_{1}$ and $P r_{2}$ represent the spatial opportunity percentages for interference $\mathrm{I}_{1}$ and $\mathrm{I}_{2}$ for the PT/SR and ST/PR, respectively. $P r_{\text {Total }}$ denotes the spatial opportunity percentage which considers both interference $\mathrm{I}_{1}$ and $\mathrm{I}_{2}$.

\section{Numerical Results}

\subsection{Simulation Condition}

In this section, we numerically evaluate the spatial opportunity for the secondary system. We evaluate the four different cases shown in Fig. 5 where path loss models (a), (b), and (c) introduced in Fig. 1 are considered. Although we consider the fixed wireless system as the primary system in Sect. 2, a mobile terminal can also be assumed as PR for the primary system in Case 1 and 2. On the other hand, we suppose that the primary system is a fixed wireless access/relay in Case 3 and 4 . The antenna height of the PR can be assumed to be high in these cases.

When considering actual deployments in the secondary system, different antenna heights must be considered. Case

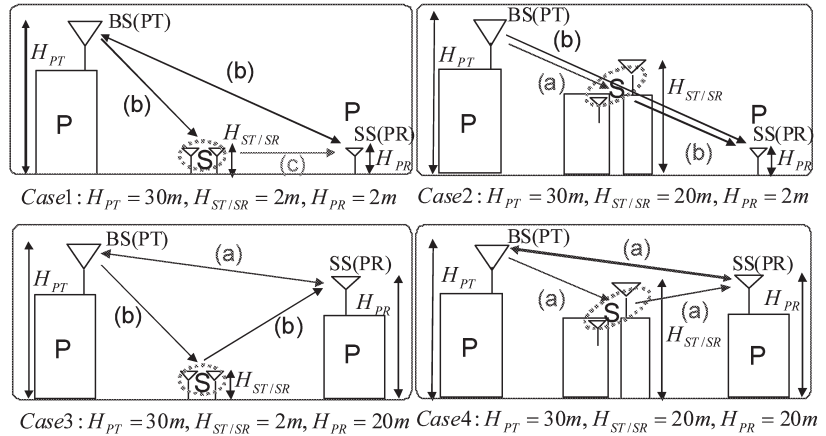

Fig. 5 Conditions including heterogeneous path loss for numerical calculations.

Table 3 Simulation parameters.

\begin{tabular}{|l|l|}
\hline Parameter & Value \\
\hline \hline Frequency, $f_{c}$ & $2.4 \mathrm{GHz}$ \\
\hline Transmit power of PT, $G_{\mathrm{PT}, \mathrm{HPA}}$ & $33 \mathrm{dBm}$ \\
\hline Transmit power of SR, $G_{\mathrm{ST}, \mathrm{HPA}}$ & $19 \mathrm{dBm}$ \\
\hline Noise power of Rx (PR and SR), $G_{\mathrm{Rx}, \mathrm{LNA}}$ & $-174 \mathrm{dBm} / \mathrm{Hz}$ \\
\hline Minimum received power of PT, $R_{\mathrm{PT}, \mathrm{PR}}\left(d_{\mathrm{PT}, \mathrm{PR}}^{\max }\right)$ & $-80 \mathrm{dBm}$ \\
\hline Minimum received power of SR, $R_{\mathrm{ST}, \mathrm{SR}}\left(d_{\mathrm{ST}, \mathrm{SR}}^{\text {max }}\right)$ & $-65 \mathrm{dBm}$ \\
\hline Target CIR & $40 \mathrm{~dB}$ \\
\hline Beamwidth of PT in E plane, $\theta_{\mathrm{PT}, \mathrm{BW}}^{v}$ & 10 degree \\
\hline Beamwidth of PR in E plane, $\theta_{\mathrm{PT}, \mathrm{BW}}^{v}$ & 30 degree \\
\hline Antenna height of PT, $H_{P T}$ & $30 \mathrm{~m}$ \\
\hline Short-term fading & Rayleigh \\
\hline Angular spread & 360 degree \\
\hline Number of trials for positions and fading conditions & $1,000,000$ times \\
\hline
\end{tabular}

1 and 3 with secondary users located at low heights ( 2 meters) represent hotspot scenarios in indoor/outdoor environments. On the other hand, since secondary users are located at high positions in Case 2 and 4, these represent cases of usage of W-LANs between buildings, e.g., relay/mesh networks.

The simulation parameters are shown in Table 3. The radio frequency is set to be $2.4 \mathrm{GHz}$, because the path loss model for the outdoor scenarios deals with only $2 \mathrm{GHz}$ band. We set the transmit power, $G_{\mathrm{Tx}, \mathrm{HPA}}$, and the minimum received power, $R_{\mathrm{Tx}, \mathrm{Rx}}^{\min }$, of both the primary and secondary systems, referring to actual systems. In this paper, $G_{\mathrm{PT}, \mathrm{HPA}}$ and $R_{\mathrm{PT}, \mathrm{PR}}^{\min }$ for the primary system are set respectively to $33 \mathrm{dBm}$ and $-80 \mathrm{dBm}$ with a $20 \mathrm{MHz}$ bandwidth, as specified by one of the Fixed Wi-Max standards [4]. For the secondary system, $G_{\mathrm{ST}, \mathrm{HPA}}$ and $R_{\mathrm{SR}, \mathrm{SR}}^{\min }$ are set respectively to $19 \mathrm{dBm}$ and $-65 \mathrm{dBm}$ with a $20 \mathrm{MHz}$ bandwidth. These values refer to IEEE802.11g systems [23]. We have set that the noise power is $-174 \mathrm{dBm} / \mathrm{Hz}(-100 \mathrm{dBm}$ at $20 \mathrm{MHz}$ bandwidth) for both primary and secondary systems. Since $R_{\mathrm{PT}, \mathrm{PR}}^{\min }=-80 \mathrm{dBm}$ and $R_{\mathrm{SR}, \mathrm{SR}}^{\min }=-65 \mathrm{dBm}, \mathrm{CNR}_{\mathrm{PT}, \mathrm{PR}}^{\min }$ and $\mathrm{CNR}_{\mathrm{SR}, \mathrm{SR}}^{\min }$ are 20 and $35 \mathrm{~dB}$, respectively.

Since the primary receiver (PR) should not be harmed by secondary transmissions, we have set the target CIR at PR equal to $40 \mathrm{~dB}$; we have chosen a level which is $20 \mathrm{~dB}$ higher than $\mathrm{CNR}$ at the $\mathrm{PR}$ (i.e., $\mathrm{CIR}=\mathrm{CNR}+20 \mathrm{~dB}=40 \mathrm{~dB}$ ). As for the secondary receiver (SR), we have set the target 


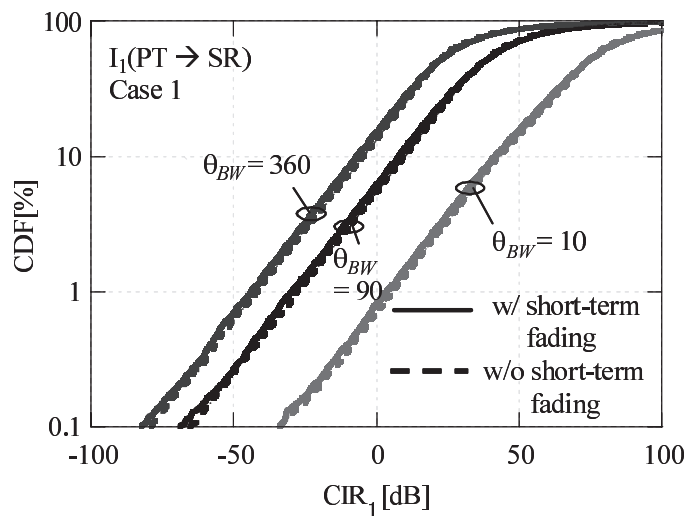

Fig. 6 Cumulative distribution function of instantaneous CIR when interference from PT to SR is considered $\left(\mathrm{I}_{1}\right)$.

CIR equal to $40 \mathrm{~dB}$. This equals the target CNR plus $5 \mathrm{~dB}$, which is less strict than PR as we take into consideration the possibility for the SR to use interference cancelation techniques [25], [26].

The antenna gain, $G_{\text {Ant }}$, can be approximately expressed as a function of the $3 \mathrm{~dB}$ antenna beamwidth in the horizontal and vertical planes $\left(\theta_{\mathrm{BW}}^{h}\right.$ and $\left.\theta_{\mathrm{BW}}^{v}[19]\right)$. The relationship among the antenna gain and the $3 \mathrm{~dB}$ antenna beamwidth in the horizontal and vertical planes can be approximately expressed as $G_{\mathrm{Ant}}=40000 / \theta_{\mathrm{BW}}^{h} \theta_{\mathrm{BW}}^{v}$. Since the antenna gain at the $\mathrm{BS}, G_{\mathrm{PT} \text {, Ant }}$, is generally higher than the one at the PR, $G_{\mathrm{PR} \text {, Ant }}$, we set the beamwidth of the PT and PR in the vertical plane to 10 and 30 degrees, respectively. When the omni-directional antennas are used for the both PT and PR, the antenna gains for the PT and PR become 11.1 and $3.7 \mathrm{dBi}$, respectively. In the cellular and/or WiMAX systems, the range of antenna gain is generally from 10 to $15 \mathrm{dBi}$ when considering the omni-directional antenna at the BS [4], [20]. Since the primary system considers a large cell, the antenna gain for PR is set to be greater than $2 \mathrm{dBi}$, which is the gain for a standard dipole omnidirectional antenna [19], even if the PR has omni-directional antenna. Hence, the values used for the beamwidth in the vertical plane are reasonable in this paper. For simplicity, the noise figures are assumed to be the same for the primary and secondary receivers.

The antenna response, $F_{\mathrm{BW}}(\phi)=\cos ^{n}(\phi)$ ( $n$ is a positive value [21]) is a function of $\theta_{\mathrm{BW}}^{h}$ [22]. To determine the sidelobe level, we impose that the integration of the $G_{\mathrm{Ant}} F_{\mathrm{BW}}(\phi)$ from 0 to $2 \pi$ should be constant regardless of the beamwidth in order to keep this model closer to the real antenna pattern. We assume flat Rayleigh fading environment with angular spread of 360 degree. The number of waves are 100 and these waves arrive uniformly from random dirtections.

\subsection{Basic Characteristics}

First, the CIR characteristics are shown in order to clarify how the interference has an impact for the secondary spatial opportunity. Figures 6 and 7 show the cumulative distribu-

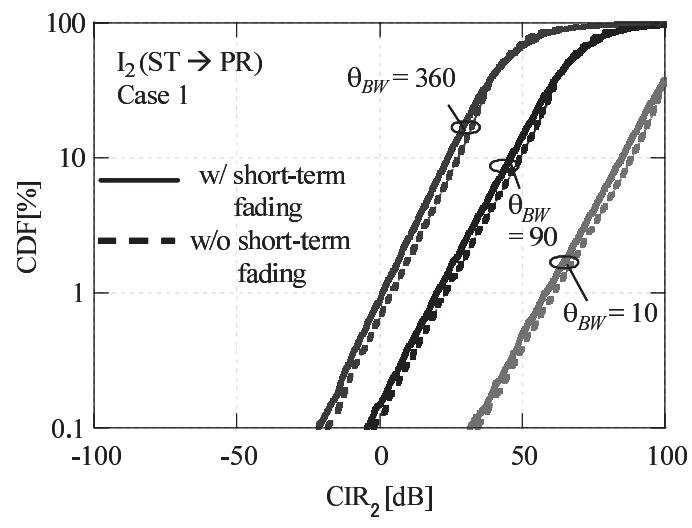

Fig. 7 Cumulative distribution function of instantaneous CIR when interference from ST to PR is considered $\left(\mathrm{I}_{2}\right)$.

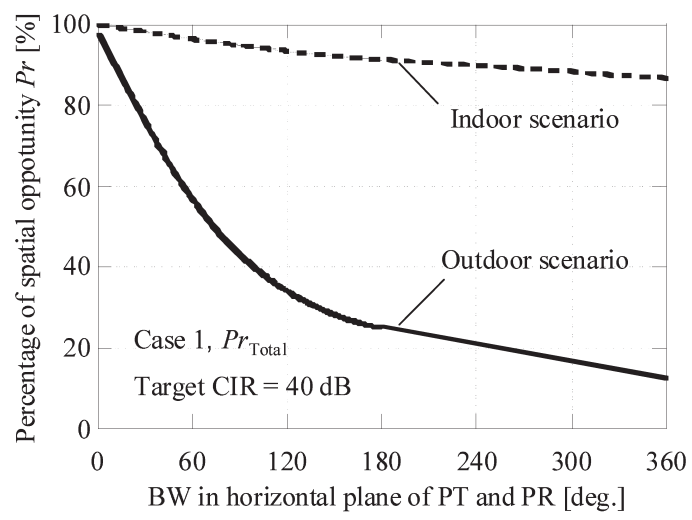

Fig. 8 Percentage for spatial opportunity when indoor and outdoor scenarios are assumed.

tion function of instantaneous CIR. Figure 6 is for the interference $I_{1}$ from PT to SR. Figure 7 is for the interference $I_{2}$ from ST to PR. We compare the influence between with and without short-term fading effects. As shown in these figures, $\mathrm{CIR}_{\mathrm{I}_{2}}$ is much larger than $\mathrm{CIR}_{\mathrm{I}_{1}}$. Moreover, the higher CIR can be guaranteed by the narrower BW in the PT and PR. For example, the probability where instantaneous $\mathrm{CIR}_{\mathrm{I}_{2}}$ is less than the target CIR $(=40 \mathrm{~dB})$ is only $0.2 \%$, when the $\mathrm{BW}$ is 10 degree. Regarding the short-term fading, the influence is much smaller than that due to the BW and path loss condition. Hence, we confirmed that the instantaneous CIR is mainly determined by the BW and the path loss condition.

Figure 8 presents the percentage spatial opportunity given in Eq. (12) versus the beamwidths (BWs) of the PT and PR. In the indoor scenario, we consider the worst case: the average distance from the wall of the building to the secondary devices is set to $40 \mathrm{~cm}$. We evaluate the percentage spatial opportunity when Case 1 in Fig. 5 is considered. As Fig. 8 clearly shows, the percentage spatial opportunity increases when the BWs of the PT and PR become narrower. This confirms that the spatial reuse is largely enhanced when directional antenna is used instead of the omnidirectional one.

Figure 8 indicates that the variations of the percentage 


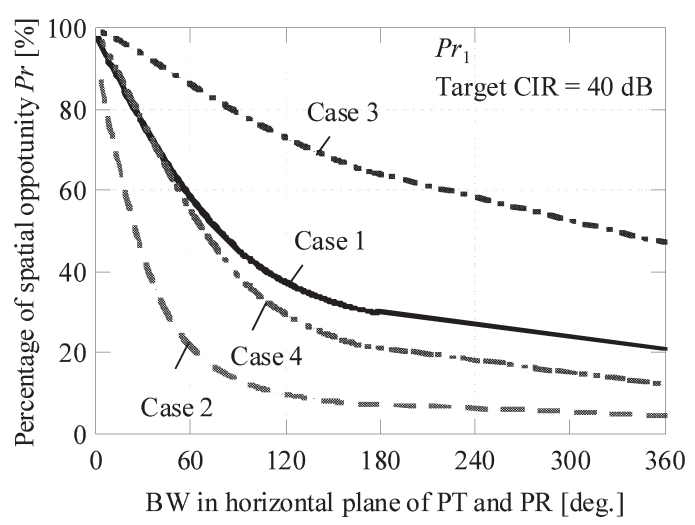

Fig. 9 Percentage for spatial opportunity when interference from PT to $\mathrm{SR}$ is considered $\left(\mathrm{I}_{1}\right)$.

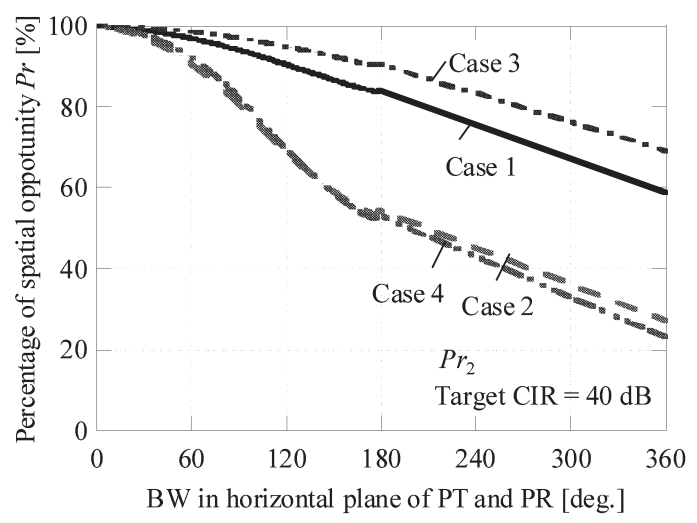

Fig. 10 Percentage for spatial opportunity when interference from ST to $\mathrm{PR}$ is considered $\left(\mathrm{I}_{2}\right)$.

the spatial opportunity versus the BW of the primary system are largely different between the indoor and outdoor scenarios. Thus, the cognitive radio in indoor scenarios demonstrates to achieve a high reuse of the spectrum on the primary system, whatever antenna pattern is applied in the primary system. In an opposite way, the directional antenna with the narrower beam is effective for the secondary system located outdoor. From this results, we assume the outdoor scenario for secondary system hereafter.

\subsection{Spatial Opportunity by Beam Width and Heteroge- neous Path Loss Conditions}

Figures 9 and 10 show the percentage for spatial opportunity versus the BW of PT and PR when Case 1 to 4 in Fig. 5 are considered. Figures 9 and 10 show the results when considering $\mathrm{I}_{1}$ (interference from PT to SR) and $\mathrm{I}_{2}$ (interference from ST to PR), respectively. As Figs. 9 and 10 clearly show, the spatial opportunity increases when the BWs of the PT and PR become narrower. This confirms that the spatial reuse is greatly enhanced when a directional antenna is used instead of the omni-directional one, when considering both interference $\mathrm{I}_{1}(\mathrm{PT} / \mathrm{SR})$ and $\mathrm{I}_{2}(\mathrm{ST} / \mathrm{PR})$.

Figure 9 shows that the absolute value of the spatial op-

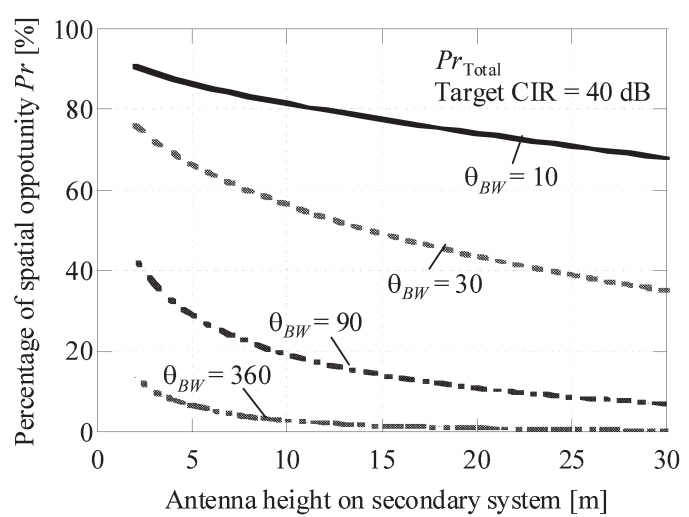

(a) $\mathrm{H}_{\mathrm{PT}}=30 \mathrm{~m}, \mathrm{H}_{\mathrm{PR}}=2 \mathrm{~m}$

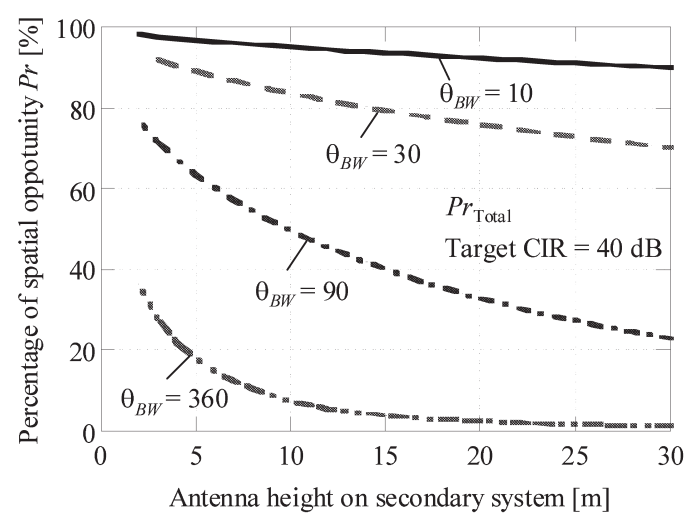

(b) $\mathrm{H}_{\mathrm{PT}}=30 \mathrm{~m}, \mathrm{H}_{\mathrm{PR}}=20 \mathrm{~m}$

Fig. 11 Percentage for spatial opportunity versus the antenna height of the secondary system.

portunity is changed among four cases. Case 3 exhibits the highest spatial opportunity percentage. The $\mathrm{CIR}_{\mathrm{I}_{1}}$ in Case 3 is higher than those in Case 2 and 4, because the path loss between the PT and SR, $L\left(d_{\mathrm{PT}, \mathrm{SR}}\right)$ in Case 3 is much larger than those in Case 2 and 4. Since communicable range in Case 3 is much larger than that in Case 1 due to the small path loss between the PT and PR in Case 3 compared to that in Case 1 , the probability where $d_{\mathrm{PT}, \mathrm{SR}}$ in Case 3 is longer than that in Case 1 is increased. Hence, the $\mathrm{CIR}_{\mathrm{I}_{1}}$ in Case 3 is higher than that in Case 1. Although the path loss conditions between the PT and SR are same between Case 2 and 4 , the probability where $d_{\mathrm{PT} \text {, SR }}$ in Case 4 is longer than that in Case 2 is increased due to the larger communicable area in Case 4 than that in Case 2. Therefore, the spatial opportunity of Case 3 is the highest of all the cases.

Next, we focus on the difference between the spatial opportunities for interference $\mathrm{I}_{1}$ (interference from PT to SR) and $\mathrm{I}_{2}$ (interference from ST to PR). As Figs. 9 and 10 clearly show, the spatial opportunity for $\mathrm{I}_{2}(\mathrm{ST} / \mathrm{PR})$ is much higher than that for $\mathrm{I}_{1}(\mathrm{PT} / \mathrm{SR})$. This result means that the transmitted interfering power from the secondary system is much smaller than received interference by the secondary system. This is due to the lower transmitting power and antenna gain of the secondary users. Therefore, we can sig- 


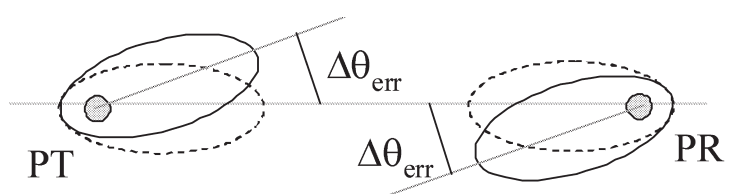

(a) Definition of error on beam pattern

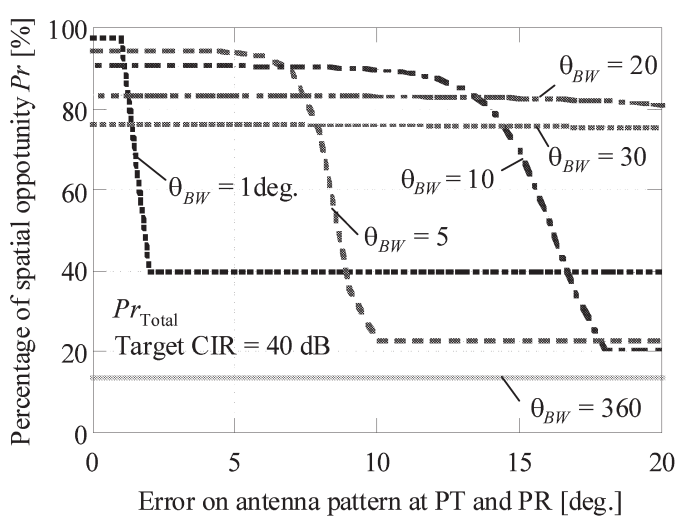

(b) Case 1

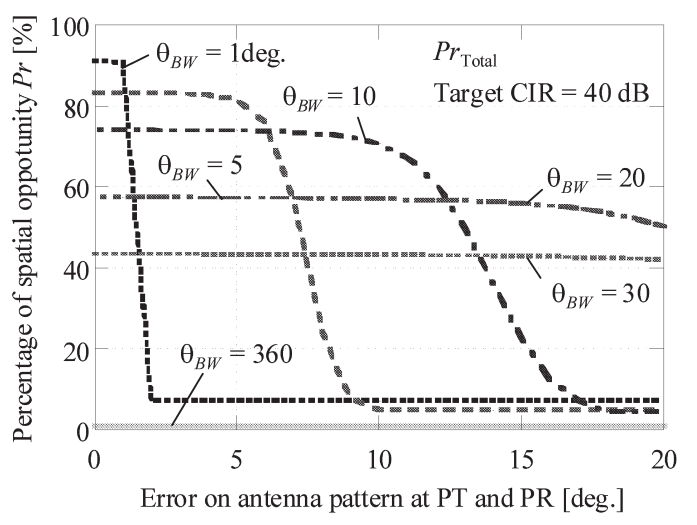

(c) Case 2

Fig. 12 Percentage for spatial opportunity when the error on the beam pattern exists.

nificantly increase the spatial opportunity for the secondary system by employing a technique to cancel the interference from the PT to the SR, which is dominant compared to that for the ST to PR [25], [26].

To understand how the difference in the antenna height for the secondary system affects the spatial opportunity more clearly, we plot the spatial opportunity versus the antenna height for the secondary system in Fig. 11. The antenna height of the PT is set to $30 \mathrm{~m}$, while the height of the PR is set to $2 \mathrm{~m}$ in Fig. 11(a) and $20 \mathrm{~m}$ in Fig. 11(b), respectively. Therefore, results in Fig. 11(a) correspond to Case 1 and 2 in Fig. 5, while the results in Fig. 11(b) correspond to those for Cases 3 and 4 in Fig. 5.

Figures 11(a) and (b) show that the spatial opportunity significantly decreases as the antenna height for the secondary system becomes higher. Figures 11(a) and (b) show the results in Case 1 and 2 in Fig. 5, respectively. In particular, the spatial opportunity is dramatically reduced when the primary system employs a broader beamwidth, be-

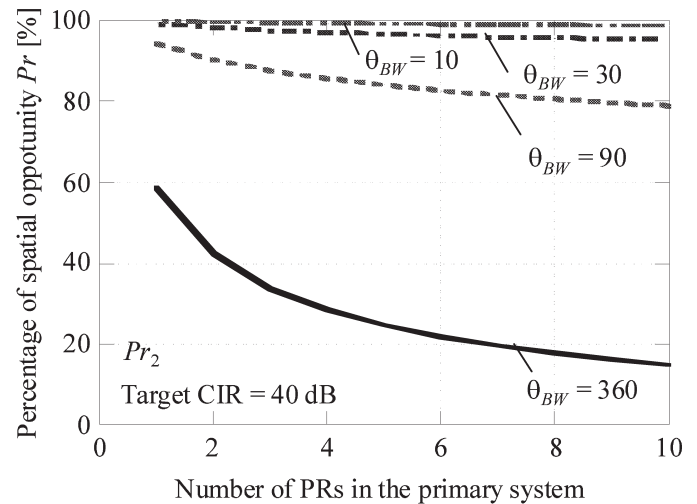

(a) Case 1

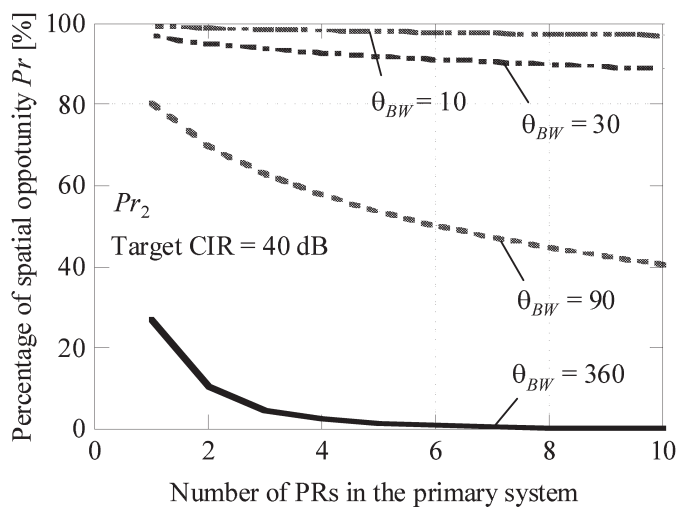

(b) Case 2

Fig. 13 Percentage for spatial opportunity versus the number of primary receivers (PRs).

cause the primary system with the broader beamwidth offers larger interference to the secondary system due to smaller path loss condition, while the primary system with the narrower beamwidth avoids the interference from/to the secondary system.

\subsection{Influence on Beam Error}

In the previous results, we assume that the beam patterns of the primary transmitter and the primary receiver align perfectly. However, the error on the main beam pattern in the primary system affects on the spatial opportunity. Figure 12 shows the spatial opportunity when the beam patterns of the primary transmitter and the primary receiver do not align perfectly. Figure 12(a) shows the definition in this evaluation. The errors from the main beam direction are give for the PT and PR, respectively. Figures 12(a) and (b) show the results in Case 1 and 2 in Fig. 5, respectively. As shown in Figs. 12(b) and (c), the percentage spatial opportunity is decreased when the error of beam pattern is approximately greater than beamwidth on the antenna pattern. However, when considering fixed wireless systems it is reasonable to assume that the error of beam patterns (Fig. 10(a)) is always smaller than beamwidth on the transmitting/receiving antennas. Therefore, we can conclude that there is higher opportunity for frequency reuse on the secondary system, when 
the primary systems are equipped with directional antennas.

\subsection{Spatial Opportunity in Multi-User Scenario in Primary System}

All previous results refer to a scenario with a primary receiver randomly located in the primary service area. Here, The spatial opportunity is investigated when multiple users are assumed in the primary system. We assume a downlink scenario in the primary system, where the BS transmits signals to the multiple SSs at the same time. Figure 13 represents the spatial opportunity percentage when multiple primary users are located within the primary service area. The results in Case 1 and 2 in Fig. 5 are presented. Figure 13 shows that the spatial opportunity is reduced by increasing the number of primary users. Particularly, the spatial opportunity is significantly decreased when using omnidirectional antennas in the primary system. A 30 to $45 \%$ reduction is observed when the omni-directional antennas are used in the primary system for Case 1 and 2 . On the other hand, the spatial opportunity is not decreased very much with a narrower beamwidth even if the number of users is increased. Therefore, co-existence can be easily achieved when directional antennas with a narrow beamwidth are employed in the primary system.

\section{Conclusion}

This paper derived the amount of spatial opportunity for secondary systems to reuse the spectrum assigned to a primary system. The proposed model can deal with both omnidirectional and directional antennas. We introduced a model that can be applied to evaluations on spatial opportunity for secondary use under various combinations of antenna heights. We demonstrated that the spatial availability when employing directional antennas can significantly enhance the opportunities of cognitive radio systems. Particularly, the reduction in the spatial opportunity is very small with directional antennas in the primary system compared to that with omni-directional antennas, even if the multi-user scenarios that generally occur in commercial systems are considered. Moreover, we clarified that different coefficients for path loss due to different antenna heights between the primary and secondary systems significantly affect the spatial opportunity for the secondary users. Such an accurate modeling of the propagation environment is key to understanding the actual benefits brought by cognitive radios.

In this paper, we focused on a primary system in which each node is in both Tx and Rx modes. Our future work is to evaluate spatial opportunity for a passive primary system where there are only nodes operating in Rx mode. The methods to estimate the exact channel state information (CSI) were not scope of study in this paper However, the CSI estimation will be a future work, especially when considering the mobility of primary users. Moreover, small-scale fading effects must be considered for more accurate evaluation.

\section{Acknowlegment}

The part of this work is supported by JSPS KAKENHI, Grant-in-Aid for Young Scientists (B) 22760272.

\section{References}

[1] Federal Communication Commission, "Spectrum policy task force," Rep. ET Docket no 02.135, Nov. 2002.

[2] S. Haykin, "Cognitive radio: Brain-empowered wireless communications," IEEE J. Sel. Areas Commun., vol.23, no.2, pp.201-202, Feb. 2005.

[3] J. Mitola, III, "Cognitive radio for flexible mobile multimedia communications," Proc. 6th International Workshop on Mobile Multimedia Communications (MoMuC), pp.3-10, Nov. 1999.

[4] X. Jing, S. Mau, D. Raychaudhuri, and R. Matyas, "Reactive cognitive radio algorithms for co-existence between IEEE $802.11 \mathrm{~b}$ and 802.16a networks," IEEE Global Telecommunications Conference (GLOBECOM'05), vol.5, pp.2465-2469, Nov./Dec. 2005.

[5] N. Hoven and A. Sahai, "Power scaling for cognitive radio," International Conference on Wireless Networks, Communications and Mobile Computing (WCNM), vol.1, pp.250-255, June 2005.

[6] R. Zhang, F. Gao, and Y. Liang, "Cognitive beamforming made practical: Effective interference channel and learning-throughput tradeoff," IEEE Trans. Commun., vol.58, no.2, pp.706-718, Feb. 2010.

[7] L. Zhang, Y. Liang, and Y. Xin, "Robust cognitive beamforming with partial channel state information," Information Sciences and Systems, 2008.

[8] S. Huang, Z. Ding, and X. Liu, "Non-intrusive cognitive radio networks based on smart antenna technology," IEEE Global Telecommunications Conference (GLOBECOM’07), 2007.

[9] K. Nishimori, E. Bottega, H. Yomo, P. Popovski, Y. Takatori, R. Prasad, and S. Kubota, "Spatial availability for cognitive radio system under directional interference," Proc. 9th International Symposium on Wireless Personal Multimedia Communications (WPMC06), San Diego, USA, Sept. 2006.

[10] K. Nishimori, R. Di Taranto, H. Yomo, P. Popovski, Y. Takatori, R. Prasad, and S. Kubota, "Spatial opportunity for cognitive radio systems with heterogeneous path loss conditions," Proc. IEEE 65th Vehicular Technology Conference (VTC2007-Spring), Dublin, Ireland, April 2007.

[11] S. Ichitsubo, T. Furono, T. Nagato, T. Taga, and R. Kawasaki, " $2 \mathrm{GHz}-$ Band propagation loss prediction in urban areas; antenna heights ranging from ground to building roof," IEICE Technical Report, A.P 96-15, May 1996.

[12] R. Di Taranto, H. Yomo, P. Popovski, K. Nishimori, and R. Prasad, "Cognitive mesh network under interference from primary user," Wirel. Pers. Commun., vol.45, no.3, pp.385-401, May 2008.

[13] M. Hata, "Empirical formula for propagation loss in land mobile radio services," IEEE Trans. Veh. Technol., vol.29, no.3, pp.317325, Aug. 1980.

[14] F. Ikegami, S. Yoshida, T. Takeuchi, and M. Umehira, "Propagation factors controlling mean field strength on urban streets," IEEE Trans. Antennas Propag., vol.32, no.8, pp.822-829, Aug. 1984.

[15] J. Walfisch and H.L. Bertoni, "A theoretical model of UHF propagation in urban environments," IEEE Trans. Antennas Propag., vol.36, no.12, pp.1788-1796, Dec. 1988.

[16] EURO-COST 231, "Urban transmission loss models for mobile radio in the 900 and 1800-MHz bands," COST 231 TD (91) 73, The Hague, The Netherlands, Sept. 1991.

[17] K. Kitao and S. Ichitsubo, "Path loss prediction formula for microcell in $400 \mathrm{MHz}$ to $8 \mathrm{GHz}$ band," Electron. Lett., vol.40, no.11, pp.685-687, May 2004.

[18] Rec. ITU-R P. 1238-4, "Propagation data and prediction methods for 
the planning of indoor radiocommunication systems and radio local area networks in the frequency range $900 \mathrm{MHz}$ to $100 \mathrm{GHz}$," ITU-R Recommendation, P Series, 2005.

[19] S.R. Saunders, Antenna and Propagation for Wireless Communication System, John Willy \& Sons, New York, 1999.

[20] Mobile WiMAX - Part I: "Technical overview and performance evaluation," Prepared on Behalf of the WiMAX Forum, Feb. 2006.

[21] K. Uehara, Y. Takatori, and K. Kagoshima, "Proposal for a novel beam combination method for indoor high-speed wireless communication systems," IEEE Trans. Veh. Technol., vol.48, no.5, pp.15111517, Sept. 1999.

[22] J.D. Kraus, Antennas, 2nd ed., McGraw-Hill, New York, 1998.

[23] IEEE802.11a, "High speed physical layer (PHY) in $5 \mathrm{GHz}$ band," 1999.

[24] Y. Okumura, E. Ohmori, T. Kawano, and K. Fukuda, "Field strength and its variability in VHF and land -mobile radio service," Rev. Elec. Commun. Lab., vol.16, pp.825-873, 1968.

[25] P. Popovski, H. Yomo, K. Nishimori, R. Di Taranto, and R. Prasad, "Opportunistic interference cancellation in cognitive radio systems," Proc. 2nd IEEE International Symposium on Dynamic Spectrum Access Networks (Dyspan2007), pp.472-475, April 2007.

[26] R. Di Taranto, K. Nishimori, P. Popovski, H. Yomo, Y. Takatori, R. Prasad, and S. Kubota, "Simple antenna pattern switching and interference-induced multi-hop transmissions for cognitive radio networks," Proc. 2nd IEEE International Symposium on Dynamic Spectrum Access Networks (Dyspan2007), pp.543-546, April 2007.

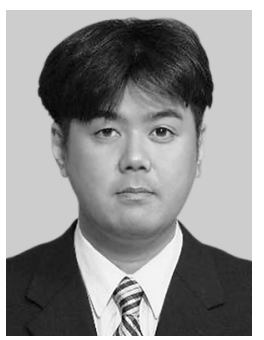

Kentaro Nishimori received the B.E., M.E. and $\mathrm{Ph} . \mathrm{D}$. degrees in electrical and computer engineering form Nagoya Institute of Technology, Nagoya, Japan in 1994, 1996 and 2003, respectively. In 1996, he joined the NTT Wireless Systems Laboratories, Nippon Telegraph and Telephone Corporation (NTT), in Japan. He was senior research engineer on NTT Network Innovation Laboratories. He is now associate professor in Niigata University. He was a visiting researcher at the Center for Teleinfrastructure (CTIF), Aalborg University, Aalborg, Denmark from Feb. 2006 to Jan. 2007. He was an Associate Editor for the Transactions on Communications for the IEICE Communications Society from May 2007 to May 2010 and Assistant Secretary of Technical Committee on Antennas and Propagation of IEICE from June 2008 to May 2010. He received the Young Engineers Award from the IEICE of Japan in 2001, Young Engineer Award from IEEE AP-S Japan Chapter in 2001, Best Paper Award of Software Radio Society in 2007 and Distinguished Service Award from the IEICE Communications Society in 2005, 2008 and 2010. His main interests are spatial signal processing including MIMO systems and interference management techniques in heterogeneous networks. He is a member of IEEE.

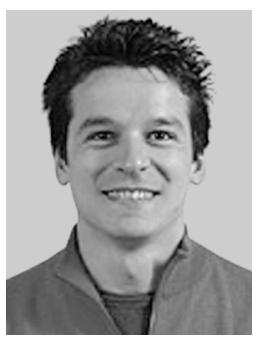

Rocco Di Taranto received the Laurea in Ingegneria delle Telecomunicazioni from Politecnico di Torino, Torino, Italy, in November 2005 and the Ph.D. Degree in Wireless Communication from Aalborg University, Denmark in June, 2010. From July 2010 to January 2011 he has worked in his village, Deliceto, Italy. Since February 2011 he is a Postdoctoral Fellow at Waterloo University, Canada. His research focuses on cognitive radio, game theory and dynamic spectrum sharing.

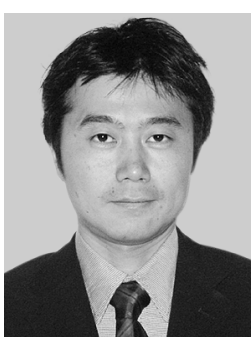

Hiroyuki Yomo received B.S., M.S., and $\mathrm{Ph} . \mathrm{D}$. degrees in communication engineering from Osaka University, Japan, in 1997, 1999 and 2002, respectively. From April 2002 to March 2004, he was a Post-doctoral Fellow at Aalborg University, Denmark. From April 2004 to September 2004, he was at NEC Corporation, Japan. In October 2004, he joined Aalborg University, Denmark, as an Assistant Research Professor, and worked as an Associate Professor from February 2006 to March 2008. From April 2008 to March 2010, he was a senior researcher at ATR, Japan. Since April 2010, he has been an Associate Professor at Kansai University, Japan. He is also affiliated with Aalborg University as an Assigned Associate Professor and with ATR as a guest researcher. He received the 2010 Funai Academic Award from Funai Foundation for Information Technology. He has received several best paper awards, including the one at IEEE Globecom 2009. His main research interests are access technologies, radio resource management, and link-layer techniques in the area of ad hoc/vehicular network, wireless LAN, cellular system, cognitive radio, and wireless network coding. He is an editor of IEEE Communications Letters.

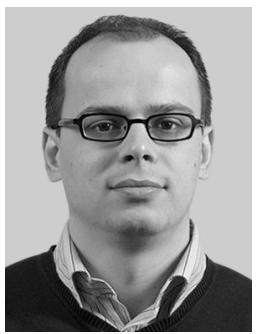

Petar Popovski received the Dipl.-Ing. in electrical engineering and M.Sc. in communication engineering from the Faculty of Electrical Engineering, Sts. Cyril and Methodius University, Skopje, Macedonia, in 1997 and 2000, respectively and a Ph.D. degree from Aalborg University, Denmark, in 2004. He worked as Assistant Professor at Aalborg University from 2004 to 2009. From 2008 to 2009 he held parttime position as a wireless architect at Oticon A/S. Since 2009, he is an Associate Professor at Aalborg University. He has more than 90 publications in journals, conference proceedings and books and has more than 20 patents and patent applications. In January 2009 he received the Young Elite Researcher award from the Danish Ministry of Science and Technology. He has received several best paper awards, among which the ones at IEEE Globecom 2008 and 2009, as well as Best Recent Result at IEEE Communication Theory Workshop in 2010. He has served as a technical program committee member in more than 30. Dr. Popovski has been a Guest Editor for special issues in EURASIP Journals and the Journal of Communication Networks. He serves on the editorial board of IEEE Transactions on Wireless Communications, IEEE Communications Letters, Ad Hoc and Sensor Wireless Networks journal, and International Journal of Communications, Network and System Sciences (IJCNS). His research interests are in the broad area of wireless communication and networking, information theory and protocol design. 